CIENCIA 17(1), 87 - 97, 2009

Maracaibo, Venezuela

\title{
Metales totales y biodisponibles en sedimentos recientes de la laguna Los Patos, estado Sucre, Venezuela
}

\author{
Aristide Márquez ${ }^{1 *}$, William Senior ${ }^{1}$, Gregorio Martínez $z^{1}$ y Ángel González ${ }^{2}$ \\ ${ }^{1}$ Departamento de Oceanografia, Instituto Oceanográfico de Venezuela, \\ Universidad de Oriente, Núcleo de Sucre. Cumaná, Venezuela \\ ${ }^{2}$ Instituto Limnológico, Universidad de Oriente, Núcleo de Bolívar
}

Recibido: 17-09-07 Aceptado 25-02-09

\section{Resumen}

El objetivo de esta investigación fue evaluar el contenido de metales totales y la biodisponibilidad de estos elementos en el sedimento de la laguna Los Patos, estado Sucre, Venezuela. Utilizando extracciones con $\mathrm{HNO}_{3}: \mathrm{HClO}_{4}: \mathrm{HCl}$ en proporción 3:1:1 para metales totales y acido acético al $10 \%$ para la fracción biodisponible, se determinó mediante técnica de espectrofotometría de absorción atómica con llama de aire acetileno que, los metales totales muestran en sus concentraciones máximas ( $\mu \mathrm{g} / \mathrm{g})$, un orden decreciente: Fe $(1304,13)>\operatorname{Mn}(177,14)>\mathrm{Zn}$ $(31,74)>\mathrm{Pb}(12,62)>\mathrm{Cu}(10,27)>\mathrm{Cr}(5,92)>\mathrm{Cd}(0,56)$, con asociación hacia las partículas de tipo lodo. La laguna presenta un deterioro con focos de contaminación por plomo en la parte central y de cobre, níquel y plomo hacia el extremo sur, lugar donde se encuentra ubicada una planta de tratamiento de aguas servidas. $\mathrm{Fe}, \mathrm{Mn}, \mathrm{Cu}, \mathrm{Zn}$ y Ni están presente en la fracción biodisponible con valores entre 2,01-99,62\%; 12,00-52,82\%; 4,53-18,88\%; 26,52-45,91\% у $2,93-91,59 \%$ respectivamente, del total de cada metal presente en el sedimento. La heterogeneidad observada en la distribución de las fracciones residual y biodisponible, refleja una gran variabilidad de la matriz sedimentaria. Por otra parte, la presencia de estos cinco metales en la fracción biodisponible evidencia un riesgo progresivo para la biota de la laguna, debido a la capacidad de estos elementos para acumularse en los organismos, sugiriendo una magnificación de estos impactos a futuro, en virtud de la débil circulación de las aguas de este ecosistema.

Palabras clave: Sedimentos, laguna, metales, biodisponibilidad.

\section{Total heavy metal and bioavailability in recent sediments from Duck' lagoon, Sucre state, Venezuela}

\begin{abstract}
The objective of this research was to assess the total metal content and bioavailability of these elements in the sediment of the Duck' lagoon (Laguna Los Patos), Sucre state, Venezuela. By using extractions with $\mathrm{HNO}_{3}: \mathrm{HClO}_{4}: \mathrm{HCl}$ in 3:1:1 ratio to total metals and acetic acid to $10 \%$ for the bioavailable fraction, was determined by atomic absorption spectrometry with air acetylene flame, that total metals show at their peak concentrations $(\mu \mathrm{g} / \mathrm{g})$, a descending order: Fe

* Autor para la correspondencia: aristd@gmail.com; aristide@sucre.udo.edu.ve
\end{abstract}


(1304.13) $>\mathrm{Mn}(177.14)>\mathrm{Zn}(31.74)>\mathrm{Pb}(12.62)>\mathrm{Cu}(10.27)>\mathrm{Cr}(5.92)>\mathrm{Cd}(0.56)$, associated with the particles of the kind of mud. The lagoons presents a deterioration with focus of lead pollution in the central part and copper, nickel and lead towards the far south, where a sewage treatment plant is located. Iron, manganese, copper, zinc and nickel are in the bioavailable fraction with values between $2.01-99.62 \%$; $12.00-52.82 \%$; $4.53-18.88 \%$; $26.52-45.91 \%$ y $2.93-91.59 \%$ respectively, of the total of each present metal in the sediment. The heterogeneity observed in the distribution of residual and bioavailable fractions, reflects a wide variability of the sedimentary matrix. Moreover, the presence of these five metals in the bioavailable fraction shows a progressive risk for biota of the lagoon, due to the ability of these elements to bioaccumulate in organisms, suggesting a magnification of these impacts in the future, under the weak flow of the waters of this ecosystem.

Key words: Sediment, lagoon, metals, bioavailability.

\section{Introducción}

Los ecosistemas marino-costeros son ambientes receptores de la mayoría de los desechos procedentes del continente y pueden ser utilizados como un indicador sensitivo en el monitoreo espacial y temporal de contaminantes como metales pesados (1). En los sedimentos, los metales pesados contienen una fracción biodisponible y acumulable para los organismos acuáticos, la cual está conformada por metales adsorbidos a la superficie de las partículas de arcilla, asociados con los carbonatos y los oxihidróxidos de hierro-manganeso reactivos, por otra parte, poseen otra fracción de tipo residual, donde el metal forma parte del retículo cristalino de las partículas del sedimento (2).

Los metales pesados son tóxicos cuando alcanzan niveles capaces de dañar las funciones vitales de un organismo. Su toxicidad en los ecosistemas depende del grado de oxidación o de la forma química en que se encuentre presente. Metales como el cadmio, cromo, cobre, hierro, níquel, plomo y de zinc exhiben toxicidad acuática cuando están en concentraciones superiores a niveles estandarizados, produciendo contaminación en los cuerpos de agua (3). En los ambientes acuáticos contaminados los organismos bioacumulan estos elementos, debido a la estabilidad química y pobre biodegrabilidad que ellos presentan. Es por ello que, el monitoreo del contenido del metales pesados en los cuerpos del agua, especialmente el derivado de la las actividades antropogénicas es una actividad particularmente importante para valoración de la calidad y protección de los ecosistemas (4).

En la laguna Los Patos actualmente muchas de las especies de aves, mamíferos, peces y crustáceos que habitaban en ella desaparecieron debido al deterioro que experimenta su ecosistema, principalmente por problemas de contaminación originado por la descarga de aguas negras provenientes de las redes cloacales de algunas urbanizaciones y del asentamiento humano en sus márgenes, lo cual ha incrementado las concentraciones de algunos compuestos de nitrógeno y fosforo en sus sedimentos (5). En virtud de las escasas investigaciones que se han realizado en el sedimento de laguna Los $\mathrm{Pa}$ tos, se plantearon como objetivos, determinar los niveles totales de algunos metales pesados como, $\mathrm{Cd}, \mathrm{Cr}, \mathrm{Cu}, \mathrm{Fe}, \mathrm{Mn}, \mathrm{Ni}, \mathrm{Pb}$ y $\mathrm{Zn}$ y cuantificar los niveles de estos elementos en la fracción biodisponible del sedimento.

\section{Materiales y métodos}

\section{Área de estudio}

La laguna Los Patos, se encuentra situada en la zona oeste de la ciudad de $\mathrm{Cu}$ maná, estado Sucre, Venezuela $(6,7)$. Forma parte de una serie de albuferas litorales comunicadas entre sí (figura 1), 


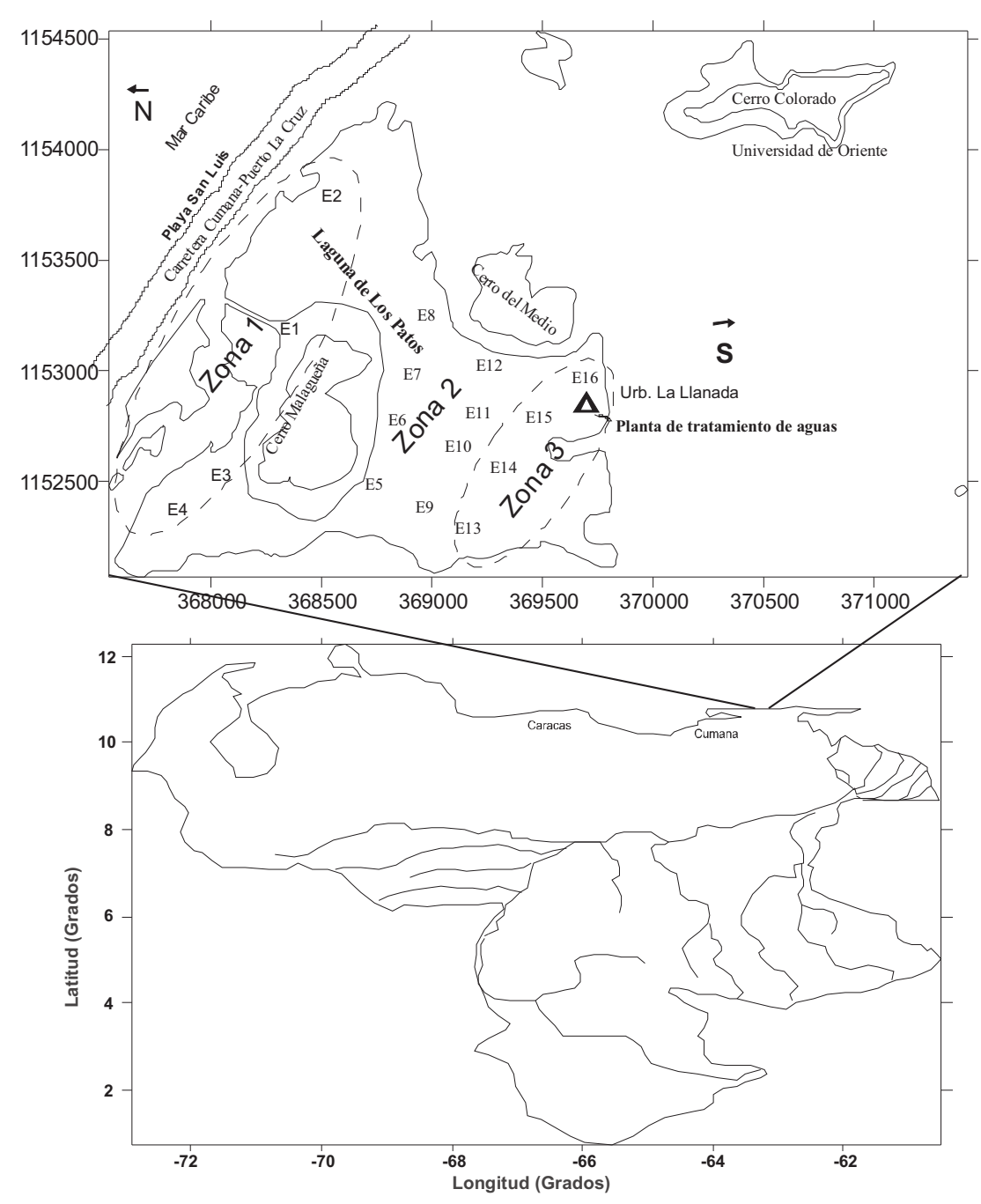

Figura 1. Zona de estudio mostrando los sitios de recolección de las muestras en la laguna Los Patos estado Sucre, Venezuela Se muestran en líneas punteadas 3 zonas dentro de la laguna; boca = zona 1 ; centro = zona 2 ; sur = zona 3.

ocupando un área total de $1,5 \mathrm{Km}^{2}$, con una profundidad que varía entre 0,40 a $1,00 \mathrm{~m}$. El fondo de la laguna es de tipo arenoso- limoso de color gris oscuro, arcilloso y de contextura gelatinosa. La laguna tiene conexión con el mar en playa San Luis; sin embargo, la acumulación de arena en la boca impide un contacto directo de las aguas durante la mayor parte del año (5). En la década de los años 40, la construcción de la carretera que comunica a las ciudades de Cumaná y Barcelona (estado Anzoátegui), produjo un dre- naje transversal insuficiente que interceptaba las aguas provenientes del drenaje natural del terreno y del excedente del sistema de riego del río Manzanares, dando origen a un cuerpo de agua con poca circulación, que induce al crecimiento de manglares en su interior (7).

\section{Recolección de muestras}

Se recolectaron dieciséis muestras de sedimento (figura 1) durante un muestreo en marzo del 2004 (época de sequía). La 
toma de muestras abarcó toda la laguna incluyendo la entrada cerca de playa San Luis, el centro de la laguna y el sector sur adyacente a la urbanización la Llanada-Autopista Antonio José de Sucre, donde está ubicada la planta de tratamiento de aguas residuales y doméstica. Para la ubicación geográfica de cada estación se utilizó un sistema de posicionamiento global (GPS) marca Garmin 12XL Los sedimentos se recolectaron utilizando una draga tipo Eckman de $0,02 \mathrm{~m}^{2}$ de área. Con el apoyo de una paleta plástica las muestras se tomaron desde la draga y se colocaron en frascos de polipropileno de $250 \mathrm{~mL}$ de capacidad previamente rotulados y lavados con una solución ácida ( $\mathrm{HCl} 1 \mathrm{~mol} / \mathrm{L})$ y agua desionizada, calidad Nanopure. Las muestras luego se preservaron bajo congelación a $-20^{\circ} \mathrm{C}$.

Para el análisis granulométrico, los sedimentos fueron secados a $80^{\circ} \mathrm{C}$ en una estufa P SELECTA (EUA) y luego Pasados través de tamices de 4,00; 2,00; 1,00; 0,85; 0,50; 0,25 y 0,063 mm. Los sedimentos preservados fueron descongelados y secados a $60^{\circ} \mathrm{C}$ en una estufa hasta obtener un peso constante, luego se tamizaron para obtener la fracción menor a $2 \mathrm{~mm}$ y se pulverizaron en un mortero de porcelana, para luego ser almacenados en envases de polietileno previamente lavados hasta los análisis.

\section{Análisis de laboratorio}

$2 \mathrm{~g}$ de muestra por triplicado fueron tratadas con una mezcla azeotrópica de $\mathrm{HNO}_{3}: \mathrm{HClO}_{4}: \mathrm{HCl}$ en proporción 3:1:1 a $110^{\circ} \mathrm{C}$, durante 30 minutos (8), en un microondas Microdigest 401 de Prolabo. Posteriormente las muestras se filtraron a través de papel de filtro Whatman con poros menores de $5 \mu \mathrm{m}$ hasta obtener un volumen de filtrado de $25 \mathrm{~mL}$.

Para el análisis de la fracción biodisponible, la cual contiene los metales adsorbidos a la superficie de las partículas de arcilla, asociados con los carbonatos y a los oxihidróxidos de manganeso reactivos, se utilizaron cuatro estaciones escogidas al azar, ubicadas en: la boca (E1), centro (E7 y E11) y sur de la laguna (E16). Para el análisis de la fracción biodisponible, la cual contiene los metales adsorbidos a la superficie de las partículas de arcilla, asociados con los carbonatos y a los oxihidróxidos de manganeso reactivos, se utilizaron cuatro estaciones escogidas al azar, ubicadas en: la boca (E1), centro (E7 y E1 1) y sur de la laguna (E16). El método utilizado para la determinación analítica fue extracción con acido acético al 25\% $(8,9)$. La cuantificación de las concentraciones se realizó por Espectrofotometría de $\mathrm{Ab}$ sorción Atómica con llama de aire-acetileno y corrección de fondo de deuterio, utilizando un equipo Perkin Elmer 3100.

Para establecer la existencia o no de contaminación por metales pesados en los sedimentos de la laguna Los Patos, se realizaron comparaciones con los niveles propuestos en el libro de texto de Sadiq (10), debido a que, uno de los principales problemas al interpretar los resultados e indicar si un sedimento está contaminado en Venezuela, es que se carece de normativas legales que señalen valores considerados como contaminantes para los sedimentos marino costeros (11). Los grados de asociación existentes entre las estaciones se determinaron por análisis estadísticos de conglomerados, empleando el método de Mínima Varianza de Ward's y la distancia métrica Euclidiana (12). Los grupos homogéneos (GH), los cuales corroboraron las diferencias estadística entre las zonas, se determinaron por la prueba de rango múltiple Student-Newman -Keuls a un nivel de significancia de $P<0,05$. Por otra parte, la asociación entre los diferentes elementos se determinó por análisis de correlación de Pearson a un nivel de $P<0,05$ (12). Los análisis computarizados de toda la estadística fue realizada utilizando el paquete estadístico Statgraphics Plus 4,1.

\section{Resultados y discusión}

El análisis de conglomerados aplicado a las concentraciones de metales obtenidas en cada estación (figura 2) y la prueba de 


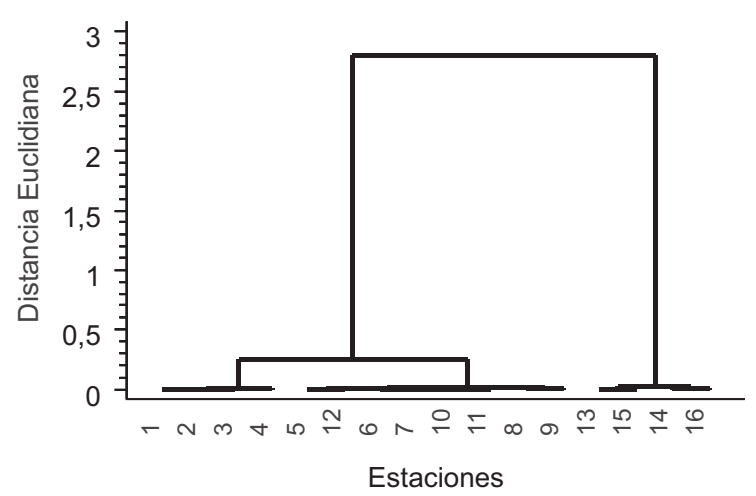

Figura 2. Dendograma de similitud que muestra la asociación entre los metales en los sedimentos recientes de la laguna Los Patos, estado Sucre, Venezuela.

rango múltiple (tabla 1) permitió identificar tres grupos zonas dentro de la laguna Los Patos. Una primera zona la conforman las estaciones: E1, E2, E3, E4 en los alrededores de la boca de la laguna. La segunda fue determinada hacia el centro de la laguna (E5, E6, E7, E8, E9, E10, E11 y E12) y la tercera situada hacia el extremo sur (E13, E14, E15y E 16), específicamente en los alrededores de la planta de tratamiento de aguas servidas de la urbanización La Llanada. La distribución del tipo de partículas dentro del sedimento mostró un incremento de partículas finas desde la boca hacia el extremo sur de la laguna. Los valores fueron 10; 26 y $64 \%$ de lodos, 55; 14 y 10\% de arena media (AM) y 35; 60 y $26 \%$ de arena fina (AF) para la boca, centro y lado sur de la laguna, respectivamente (tabla 2). Los promedios de las concentraciones de metales pesados determinados para cada una de estas tres zonas, fueron utilizados para hacer la interpretación de los resultados del ecosistema bajo estudio.

Las concentraciones máximas de metales ( $\mu \mathrm{g} / \mathrm{g})$ en los sedimentos de la laguna Los Patos (tabla 2) variaron en orden decreciente: $\mathrm{Fe}(1304,13)>\operatorname{Mn}(177,14)>\mathrm{Zn}$ $(31,74)>\mathrm{Pb}(12,62)>\mathrm{Cu}(10,27)>\mathrm{Cr}(5,92)$
$>$ Cd $(0,56)$. Por otra parte, la tendencia de las distribuciones de los valores, mostró un gradiente creciente desde la boca hacia el extremo sur de la laguna, con asociación hacia las partículas más finas como los lodos (figura 3). La presencia de cadmio solo fue determinada en la parte central y en el lado sur de la laguna, en donde las concentraciones alcanzaron valores de $0,037 \mu \mathrm{g} / \mathrm{g}$ y $0,56 \mu \mathrm{g} / \mathrm{g}$ respectivamente, no detectándose este elemento en la fracción biodisponible. Roux et al. (13) señalan que el cadmio se asocia a los carbonatos en proporciones superiores a $50 \%$ cadmio se encuentra asociado a los carbonatos, y otra importante proporción se encuentra asociada a la fracción intercambiable. Cuando el contenido en los sedimentos de un ecosistema sobrepasa la concentración de $1 \mu \mathrm{g} / \mathrm{g}$ los sedimentos presentan efectos de contaminación (5). En este contexto, no se evidencia contaminación por este metal en la laguna de los Patos. Adriano (2) indican que, una fuente de cadmio son las aguas municipales, sin embargo, Méndez-García et al. (14), señala que el cadmio es uno de los metales pesados con mayor grado de solubilidad, que podría asimilarse por las plantas lixiviarse hacia los acuiferos si las condiciones son propicias y que el cadmio presenta un sistema de regeneración parecido a la materia orgánica, por lo que la surgencia afecta sus concentraciones igual que los nutrientes. En la zona costera de Baja California (México) se ha observado que mientras la surgencia contribuye con 99\% de cadmio hacia la costa, 1\% es aportado por las aguas residuales (15). Por otra parte, Rahmani (16) indica que las aguas residuales son una fuente importante de Cd, y que su utilización para el riego en la agricultura incrementa la bioacumulación en las plantas, tal como ocurre en la provincia de Esfahan, Irán.

Las concentraciones de cromo alcanzaron 1,$68 ; 2,09$ y $5,92 \mu \mathrm{g} / \mathrm{g}$ en la boca, centro y lado sur, respectivamente. Sadiq (10) señala que, en los sedimentos no contamina- 
Tabla 1

Concentración de metales pesados totales $(\mu \mathrm{g} / \mathrm{g})$ y porcentajes de partículas en sedimentos recientes de la laguna Los Patos, estado Sucre, Venezuela.

\begin{tabular}{|c|c|c|c|c|c|}
\hline \multicolumn{3}{|c|}{$\mathrm{AF}$} & \multicolumn{3}{|c|}{$\mathrm{AM}$} \\
\hline Zona & Prom & GH & Zona & Prom & $\mathrm{GH}$ \\
\hline Boca & 35 & $X$ & Boca & 55 & $X$ \\
\hline Centro & 60 & $X$ & Centro & 14 & $X$ \\
\hline Sur & 26 & $X$ & Sur & 10 & $X$ \\
\hline \multicolumn{3}{|c|}{ Lodos } & \multicolumn{3}{|c|}{$\mathrm{Cd}$} \\
\hline Zona & Prom & GH & Zona & Prom & $\mathrm{GH}$ \\
\hline Boca & 10 & $\mathrm{X}$ & Boca & $<\mathrm{LD}$ & $\mathrm{X}$ \\
\hline Centro & 26 & $\mathrm{X}$ & Centro & 0,037 & $\mathrm{X}$ \\
\hline Sur & 64 & $\mathrm{X}$ & Sur & 0,56 & $X$ \\
\hline \multicolumn{3}{|c|}{$\mathrm{Cr}$} & \multicolumn{3}{|c|}{$\mathrm{Cu}$} \\
\hline Zona & Prom & GH & Zona & Prom & $\mathrm{GH}$ \\
\hline Boca & 1,68 & $\mathrm{X}$ & Boca & 1,12 & $\mathrm{X}$ \\
\hline Centro & 2,09 & $\mathrm{X}$ & Centro & 4,09 & $\mathrm{X}$ \\
\hline Sur & 5,92 & $\mathrm{X}$ & Sur & 10,27 & $\mathrm{X}$ \\
\hline \multicolumn{3}{|c|}{$\mathrm{Fe}$} & \multicolumn{3}{|c|}{$\mathrm{Mn}$} \\
\hline Zona & Prom & GH & Zona & Prom & $\mathrm{GH}$ \\
\hline Boca & 1283,24 & $\mathrm{X}$ & Boca & 7,80 & $\mathrm{X}$ \\
\hline Centro & 1287,62 & $X$ & Centro & 44,65 & $\mathrm{X}$ \\
\hline Sur & 1304,13 & $X$ & Sur & 177,14 & $\mathrm{X}$ \\
\hline \multicolumn{3}{|c|}{$\mathrm{Ni}$} & \multicolumn{3}{|c|}{$\mathrm{Pb}$} \\
\hline Zona & Prom & $\mathrm{GH}$ & Zona & Prom & $\mathrm{GH}$ \\
\hline Boca & 4,46 & $\mathrm{X}$ & Boca & 0,84 & $\mathrm{X}$ \\
\hline Centro & 7,05 & $X$ & Centro & 6,00 & $\mathrm{X}$ \\
\hline Sur & 14,30 & $\mathrm{X}$ & Sur & 12,62 & $X$ \\
\hline \multicolumn{3}{|c|}{$\mathrm{Zn}$} & & & \\
\hline Zona & Prom & GH & & & \\
\hline Boca & 7,06 & $\mathrm{X}$ & & & \\
\hline Centro & 15,46 & $\mathrm{X}$ & & & \\
\hline Sur & 31,74 & $X$ & & & \\
\hline
\end{tabular}

Prom $=$ promedio $. \mathrm{GH}=$ grupos homogéneos. $\mathrm{AF}=$ área fina. $\mathrm{AM}=$ arena media $. \mathrm{LD}=$ límite de detección .

Tabla 2

Límite de detección del método de análisis utilizado para la cuantificación de los metales pesados en los sedimentos de la laguna Los Patos, estado Sucre, Venezuela. (Metales en concentración de ( $\mu \mathrm{g} / \mathrm{g}$ ).

\begin{tabular}{ccccccccc}
\hline Metales & $\mathrm{Cd}$ & $\mathrm{Cr}$ & $\mathrm{Cu}$ & $\mathrm{Fe}$ & $\mathrm{Ni}$ & $\mathrm{Mn}$ & $\mathrm{Pb}$ & $\mathrm{Zn}$ \\
\hline Límite de detección & 0,004 & 0,02 & 0,02 & 0,01 & 0,004 & 0,002 & 0,001 & 0,002 \\
\hline
\end{tabular}




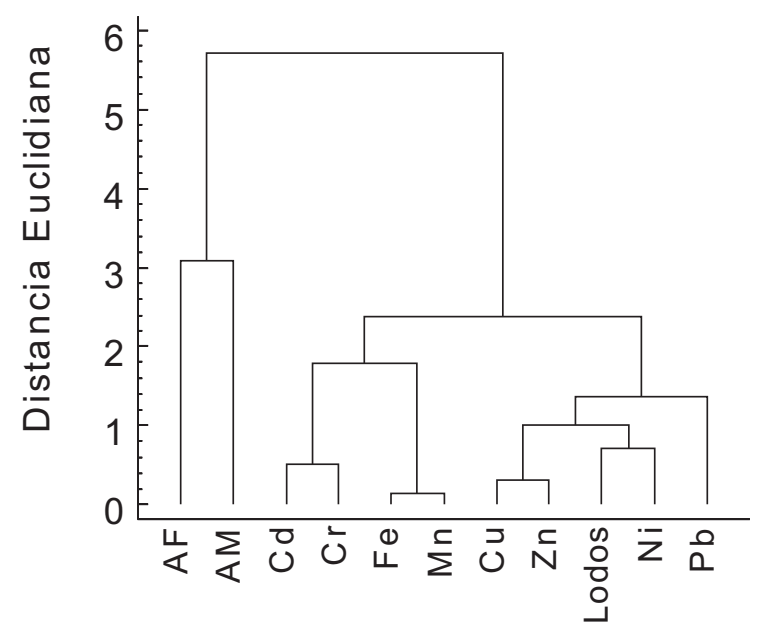

Figura 3. Dendograma de similitud obtenido con el análisis de conglomerado, mostrando las asociaciones entre la granulometría y las concentraciones de los metales totales en la laguna de Los Patos, estado Sucre, Venezuela.

dos los valores de cromo son inferiores $20 \mu \mathrm{g} / \mathrm{g}$. Los resultados obtenidos en esta investigación son inferiores a las concentraciones señaladas por este autor, no evidenciándose contaminación por cromo en la laguna de Los Patos. Por otra parte no se detectó cromo en la fracción biodisponible al menos en las cuatro estaciones utilizadas para la el estudio de biodisponibilidad. Estas observaciones han sido evidenciadas para otros ecosistemas venezolanos como la laguna de Píritu en donde, el cromo se encuentra principalmente en forma residual, siendo exclusivamente su origen de tipo litogénico (17). Los valores de cobre oscilaron entre $1,12 \mu \mathrm{g} / \mathrm{g}$ en la boca hasta $10,27 \mu \mathrm{g} / \mathrm{g}$ en el extremo sur, con valores intermedios de 4,09 $\mu \mathrm{g} / \mathrm{g}$ en el centro. La fracción biodisponible de cobre dentro de la laguna fue heterogénea (tabla 3), determinándose porcentajes de $14,57 \%$ del total de cromo presente en el sedimento en las inmediaciones de la boca específicamente en $\mathrm{E} 1$, mientras que hacia el centro (E7 y E11) los porcentajes alcanzaron 7,01 y $18,88 \%$, respectivamente.
Las menores cantidades fueron observadas hacia la parte sur donde se obtuvieron valores de $4,53 \%$. Si se consideran las concentraciones de cobre señalados por Sadiq (10) para sedimentos no contaminados $1<10$ $\mu \mathrm{g} / \mathrm{g}$ ), se podría inferir que, la Laguna Los Patos solo presenta focos de contaminación en la parte sur del sistema.

Adriano (2) señala que, la composición de las diferentes especies de $\mathrm{Cu}$ depende del $\mathrm{pH}$ y de la presencia en el agua de otros ligandos orgánicos e inorgánicos y que a niveles de pH y anhídrido carbónico característicos de aguas naturales frescas, la mayor parte del cobre soluble se presenta como complejos de carbonato cúprico. Sin embrago a altas concentraciones es tóxico para organismos acuáticos como, crustáceos, anélidos, peces y plantas. Una fuente potencial de contaminación antropogénica de cobre lo constituye los desechos sólidos de los rellenos sanitarios y las aguas las aguas servidas, los cuales contribuyen a las emisiones posteriores hacia las aguas de ríos, quebradas y lagunas, concentrándose en los sedimentos por adsorción o precipitación, tal como ocurre en el Distrito Río Azul, Costa Rica (18).

Las concentraciones de hierro mostraron un gradiente decreciente desde el extremo sur $(1304,13 \mu \mathrm{g} / \mathrm{g})$ hasta la boca $(1283,24 \mu \mathrm{g} / \mathrm{g})$. En el centro los valores fueron intermedios $(1287,62 \mu \mathrm{g} / \mathrm{g})$. Mediante el estudio de biodisponibilidad del hierro (tabla 1) se determinó que, la fracción biodisponible es variable en algunas zonas de la laguna, detectándose porcentajes de $2 \%$ en la estación $\mathrm{E} 1$, mientras que para E7, E11 y E16, los valores para esta fracción fueron de $99,62 \%, 67,89 \%$ y $45,44 \%$, evidenciando posibles diferencias en la sedimentología de la laguna. El origen del hierro en la laguna Los Patos es variable, puesto que dos de las cuatro estaciones utilizadas para el fraccionamiento muestran altos porcentajes de hierro residual que sobrepasan un 50\% (E16 y E1). Esto estaría sugiriendo un ori- 
gen litogénico. Sin embargo, lo apreciado en las estaciones E7 y E11 muestran que, parte del hierro presente en los sedimentos puede tener origen antropogénico y a la vez pueden ser incorporados con facilidad a la columna de agua y trasladados posteriormente a las plantas (19) y al resto de la cadena trófica dada su tendencia a formar complejos órgano-metálicos, lo que facilita su solubilidad, disponibilidad (20).

Las concentraciones promedios de manganeso mostraron un gradiente creciente desde la boca hasta el extremo sur con valores de 7,80; 44,65 $\mu \mathrm{g} / \mathrm{g}$ y 177,14 $\mu \mathrm{g} / \mathrm{g}$ en la boca, centro y lado sur, respectivamente. El fraccionamiento indicó que $52,82 \%$ de manganeso biodisponible se encuentra hacia la boca de la laguna, valores que descienden hasta $33,25 \%$ en E7 y a $12 \%$ y $16,44 \%$ en E11 y E16, respectivamente (tabla 2). Wollast et al. (21) señalan que, el manganeso se encuentra en una alta proporción en forma de óxidos. Esta situación no es evidenciada con claridad en la laguna Los Patos, debido a que se detectaron solo porcentajes superiores a 50\% manganeso intercambiable en la estación 1 (E1). Estas observaciones sugieren un grado de composición altamente variable en la matriz sedimentaria de los sedimentos de esta laguna.

Los niveles de níquel se incrementaron desde la boca hacia el extremo sur con valores de 4,46; 7,06 y 14, $30 \mu \mathrm{g} / \mathrm{g}$ en la boca, centro y lado sur, respectivamente. La fracción biodisponible de níquel (tabla 3) fue muy variable, evidenciado por la discrepancia de los porcentajes en cada una de las estaciones evaluadas. Se observaron porcentajes de $31,43 \%$ en la estación 1, mientras que para la estación 7 estos valores se incrementaron en dos unidades hasta 33,25\%. El mínimo y el máximo en los porcentajes de níquel biodisponible se detectaron en E16 $(2,93 \%)$ y en E1 $1(91,59 \%)$. Sadiq (10) señala que, los sedimentos no contaminados presentan valores inferiores de $10 \mu \mathrm{g} / \mathrm{g}$, de acuerdo a los resultados obtenidos solo la zona sur presenta evidencia alteración de los sedimentos, probablemente de origen antropogénico. El níquel transportado en forma suspendida por las aguas y que posteriormente entra al sedimento posiblemente es en su mayoría de tipo litogénico, ya que está asociado a minerales de arcilla, especialmente aluminosilicatos (22).

Las concentraciones de plomo mostraron un gradiente creciente desde la boca hasta el extremo sur con valores intermedios hacia el centro de la laguna. Los valores determinados para las tres zonas fueron $0,84 \mu \mathrm{g} / \mathrm{g}$ en la boca; $6 \mu \mathrm{g} / \mathrm{g}$ en el centro y $12,62 \mu \mathrm{g} / \mathrm{g}$ en el lado sur. El plomo en forma biodisponible en los sedimentos de la laguna de Los Patos, no fue detectado por la técnica de absorción atómica de llama utilizada, debido a que las concentraciones estar por debajo del límite de detección, al igual a lo observado para metales como el $\mathrm{Cr}$ y $\mathrm{Cd}$ (tabla 2). Esta última observación es diferente a la reportada para los sedimentos de otras lagunas venezolanas, como la laguna de Píritu en donde López et al. (17) indican

Tabla 3

Porcentajes (\%) metales biodisponibles en los sedimentos de la laguna Los Patos, estado Sucre, Venezuela.

\begin{tabular}{lrrrrc}
\hline & $\mathrm{Cu}$ & $\mathrm{Fe}$ & $\mathrm{Mn}$ & $\mathrm{Ni}$ & $\mathrm{Zn}$ \\
\hline E1 & 14,57 & 2,01 & 52,82 & 31,43 & 45,91 \\
E7 & 7,01 & 99,62 & 33,25 & 20,21 & 43,48 \\
E11 & 18,88 & 67,89 & 12,00 & 91,59 & 28,94 \\
E16 & 4,53 & 45,44 & 16,44 & 2,93 & 26,52 \\
\hline
\end{tabular}


que, parte del plomo presente en los sedimentos se encuentra en la fracción intercambiable y disponible para los organismos. Sadiq (10) indica que, las actividades humanas son las fuentes principales de la contaminación por este elemento y que niveles inferiores a $5 \mu \mathrm{g} / \mathrm{g}$ son considerados como no contaminantes. De acuerdo a estas observaciones se evidencia contaminación por plomo en el centro de la laguna, con un gradiente creciente hacia el extremo sur. Las concentraciones de Plomo determinados en esta investigación son inferiores a los valores entre 21,7 y $68,9 \mu \mathrm{g} / \mathrm{g}$ reportados por Frías-Espericueta et al. (23) para los sedimentos de la laguna Huizache-Caimanero, y a las concentraciones (46-294 $\mu \mathrm{g} / \mathrm{g}$ ) del sistema lagunar Altata-Ensenada Pabellón, México (24).

Los niveles de zinc decrecen desde el lado sur $(31,75 \mu \mathrm{g} / \mathrm{g})$ hasta la boca $(7,06 \mu \mathrm{g} / \mathrm{g})$, con valores intermedios de $15,46 \mu \mathrm{g} / \mathrm{g}$ en el centro. Se determinaron porcentajes variables en la fracción biodisponible de zinc en las estaciones estudiadas, detectándose porcentajes de $45,91 \%$ en E1 y $43,48 \%$ en E7. E11 y E16 mostraron valores de $28,94 \%$ y $26,52 \%$ respectivamente (tabla 4). Sadiq (10) señala que, concentraciones de zinc inferiores a $110 \mu \mathrm{g} / \mathrm{g}$ corresponden a sedimentos no contaminados, valores que son superiores a los encontrados en la laguna Los Patos. De acuerdo a esta observación no se evidencia contaminación por zinc. Sin embargo, la presencia de zinc en la fracción biodisponible es un factor desfavorable para la biota presente en la laguna Los Patos debido a que la biodisponibilidad del zinc ha sido documentada en investigaciones realizadas con plantas, peces, organismos bentónicos e invertebrados que se alimentan de los sedimentos $(25,26)$. Márquez et al. (11) reportaron para los sedimentos de la laguna de Unare en Venezuela, valores altos $(217,49 \mu \mathrm{g} / \mathrm{g})$, así como evidencias de bioacumulación en las diferentes especies de peces y crustáceos que habitan en esta laguna.
Las concentraciones de metales presentaron correlaciones estadísticas significativamente altas con los lodos ( $r>0,90$; $\mathrm{P}<0,05$, figura 3) y con el hierro. Rubio y col. (27) señalan que, la correlación que muestran en algunos metales pesados con el hierro indica fuentes similares. Estos señalamientos son evidenciados en la presente investigación, por la alta correlación significativa de todos los metales con el hierro; $\mathrm{Cu}$ $(r=0,86), \mathrm{Mn}(\mathrm{r}=0,95) ; \mathrm{Ni}(\mathrm{r}=0,74) ; \mathrm{Zn}$ $(r=0,86), \mathrm{Cd}(\mathrm{r}=0,95) ; \mathrm{Pb}(\mathrm{r}=0,95) ; \mathrm{Cr}$ $(r=0,92)$. Estos autores afirman que la correlación de los metales pesados con el hierro debe ser el comportamiento esperado en condiciones naturales ya que el hierro es un elemento definitorio de las características de los sedimentos, razón por la cual la relación del hierro con cualquier otro metal tiende a formar una tendencia lineal, lo contrario podría ser significativo de una contaminación de tipo antrópico. Tomando en consideración esta observación y los resultados obtenidos del estudio de biodisponibilidad, se podría inferir que la mayoría de los metales evaluados en esta investigación, tienen orígenes similares, sin embargo, el cadmio y cromo estarían asociados al hierro en forma diferente a oxihidróxidos debido a que, no se encontraron presentes en la fracción biodisponible.

\section{Conclusiones}

La laguna Los Patos muestra un evidente impacto por metales pesados con una asociación hacia las partículas del sedimento tipo lodo. Las concentraciones de estos elementos dentro de la laguna presentan en forma general, un gradiente de creciente desde la boca hacia el extremo sur donde se encuentra la planta de tratamiento de las aguas servidas. Se observaron focos de contaminación por cobre, níquel y plomo hacia el extremo sur y por plomo en la parte central de la laguna, sugiriendo probables aportes desde las aguas que son vertidas a la laguna desde la planta de tratamiento. Estas acumulaciones de metales en el sedimento, 
a futuro probablemente se incrementarán debido a la escasa circulación que presenta el agua de este ecosistema. La mayoría de los metales estudiados muestran asociaciones con los lodos y orígenes similares que podrían ser, asociado a compuestos como oxihidróxidos de hierro, sin embargo, el cadmio y cromo estarian asociados principalmente al hierro en forma diferente a oxihidróxidos de ese metal por no encontrarse presentes en la fracción biodisponible. A pesar de esta observación, se apreció que los sedimentos de la laguna presentan una matriz sedimentaria bastante variada y compleja, en donde se alternan en proporciones variables las formas biodisponible y residual. Esto se observa especialmente para hierro, manganeso, cobre, zinc, níquel, no teniéndose una explicación para este fenómeno. La presencia de estos cinco metales en la fracción biodisponible los hace aprovechables y bioacumulables para la biota que habita en la laguna Los Patos, evidenciando un peligro potencial para este ecosistema en el presente y en el futuro.

\section{Referencias bibliográficas}

1. BALLS P., HULL S., MILlER B., PIRIE J., PROCTOR W. Mar Poll Bull 34(1): 42-50. 1997.

2. ADRIANO D. Biogeochemistry of trace metals. Lewis Publisher (eds.), Boca Raton. USA. 513 pp. 1992.

3. EMOYAN O., OGBAN F., AKARAH E. J Appl Sci Environ Mgt 10(2): 121-127. 2006

4. PRIJU C., NARAYANA A. Int $\boldsymbol{J}$ Environ Res 1(4): 280-289. 2007.

5. MÁRQUEZ A., SENIOR W., MARTÍNEZ G., GONZÁLEZ A. Bol Inst Oceanogr Univ Oriente 46(2): 133-141. 2007.

6. LAREZ L. Lagena 11: 13-19. 1966.

7. RAMÍREZ I., ROA MORALES P. Manejo $\boldsymbol{y}$ aprovechamiento acuicola de lagunas costeras en América Latina y el Caribe. Proyecto Aquila II. GCP/RLA/102/ITA. México. Pág. 336. 1994.
8. LANDING W., LEWIS B. Marine Particles: Analysis and Characterization. (Eds. Hurd D. \& Spencer D.). American Geographysical Union. Washington D.C. (USA). 263-272. 1991.

9. LEWIS B., LANDIG W. Mar Chem 40: 10514. 1992.

10. SADIQ M. Marine Environments. Marcel Dekker Inc. (eds.). New York (USA). Pag. 389. 1992.

11. MÁRQUUEZ A., SENIOR W., FERMÍN I., MARTÍNEZ G., CASTAÑEDA J., GONZÁLEZ A. Rev Cient FCV-LUZ XVIII(1): 1-14. 2008.

12. SOKAL R., ROHLF J. Biometry. W.H. Freeman \& Company. New York (USA). 776 pp. 1969.

13. MARTÍNEZ G., SENIOR W., MÁRQUEZ A. Bol Inst Oceanogr Univ Oriente 44(2): 75-87., 2005.

14. MÉNDEZ-GARCÍA T., RODRÍGUEZDOMÍNGUEZ I., PALACIOS-MAYORGA S. Terra 18(4): 277-288. 2000.

15. GUTIÉRREZ-GALINDO E., MUÑOZ BARBOSA A. Cienc Mar 27(2): 269-288. 2001.

16. RAHMANI H. Sci Res and Essay 2(3): 8488. 2007.

17. LÓPEZ F., SENIOR W., FERMÍN I., MÁRQUEZ A., MARTÍNEZ G. Bol Inst Oceanogr Univ Oriente 45(1): 51-60. 2006.

18. MORA R., MORA R. Ambientales 29: 6375. 2005.

19. NAVARRO-AVIÑÓ J. Ecosistemas 16(2): 10-25. 2007.

20. PÉREZ M., MARTÍNEZ G., FERMÍN I. Bol Inst Oceanogr Univ Oriente 45(2): 81-91. 2006.

21. WOLLAST R., BILLEN G., DUINKER J. Estuar Coast Mar. Sci. 8(1): 161-169., 1979.

22. ROUX L., LE ROUX S., APPRIOU P. Mar Poll Bull 36(1): 56-64. 1998.

23. FRÍAS-ESPERICUETA M., OSUNA-LÓPEZ J., LÓPEZ-SÁENZ P., LÓPEZ-LÓPEZ G., IZAGUIRRE-FIERRO G. Bull Environ Contam Toxicol 73: 749-755. 2004. 
24. GREEN-RUIZ C., PÁEZ OSUNA F. Bull Environ Contam Toxicol 71: 52-59. 2003.

25. SHCUMATCHER M., DOMINGO J., LLOBET J., CORBELLA J. J Envir Sci and Health 30(6): 1361-1372. 1995.
26. VERRENGIA-GUERRERO N., KESTEN E. Envir Toxic and Water Qual 8(1): 335344. 1993.

27. RUBIO B., NOMBELA M., VILAS F. Mar Poll Bull 40(11): 968-980. 2000. 